\title{
NORMS ON ENVELOPING ALGEBRAS
}

\author{
DENIS LUMINET
}

\begin{abstract}
Let $\mathfrak{g}$ be a complex Lie algebra, and let $U$ be its enveloping algebra. $U$ is normable if and only if $\mathfrak{g}$ is nilpotent.
\end{abstract}

Let $A$ be a complex algebra. Then the following are clearly equivalent:

(1) $A$ has an algebra norm.

(2) $A$ can be embedded into a Banach algebra.

(3) $A$ admits a faithful representation by bounded Banach space operators.

Several authors $[\mathbf{1}, \mathbf{3}]$ studied the normability problem for $A$ commutative. We consider here the case $A=U(\mathfrak{g})$, where $\mathfrak{g}$ is a Lie algebra. When $\mathfrak{g}$ is abelian, $U(\mathfrak{g})$ is a polynomial algebra, which is normable. On the other hand, when $\mathfrak{g}$ is semisimple, it is not difficult to show that $U(\mathfrak{g})$ has no submultiplicative norm (see $[\mathbf{4}, \mathbf{5}])$.

Our main result is the following

THEOREM. $U(\mathfrak{g})$ has an algebra norm if and only if $\mathfrak{g}$ is nilpotent.

Proof. (1) Only if. Assume $\mathfrak{g}$ is not nilpotent. By Engel's theorem, there exists $x \in \mathfrak{g}$ such that ad $x: \mathfrak{g} \rightarrow \mathfrak{g}$ is not nilpotent, so ad $x$ has a nonzero eigenvalue $\lambda$ with nonzero eigenvector $y$. This means that $[x, y]=\lambda y$. Now there cannot be a submultiplicative norm \|\| on $U(\mathfrak{g})$, for $x y-y x=\lambda y$ implies $x y^{k}-y^{k} x=k \lambda y^{k}$ $(k \in \mathbf{N})$, whence $2\|x\|\left\|y^{k}\right\| \geq k|\lambda|\left\|y^{k}\right\|$ and $k \leq 2\|x\| /|\lambda|$ for all $k$, a contradiction.

(2) If. Suppose $\mathfrak{g}$ is nilpotent. Then [2, Theorem 2.5.5] $\mathfrak{g}$ can be embedded as a subalgebra of $\mathfrak{n}$, the Lie algebra of strictly triangular $n \times n$ matrices (for some $n$ ). Since $U(\mathfrak{g})$ embeds into $U(\mathfrak{n})$, it will be sufficient to show that $U(\mathfrak{n})$ has a faithful representation by bounded operators on a Banach space.

We consider the Lie algebras

$$
\begin{aligned}
& \mathfrak{s}=\mathfrak{g l}(n, C), \\
& \mathfrak{r}=\left\{x \in \mathfrak{s}:(x)_{i j}=0 \text { for } i>j\right\}, \text { and } \\
& \mathfrak{n}=\left\{x \in \mathfrak{s}:(x)_{i j}=0 \text { for } i \geq j\right\}=[\mathfrak{r}, \mathfrak{r}] .
\end{aligned}
$$

Let $U(\mathfrak{s})=\bigoplus_{d=0}^{\infty} U^{d}(\mathfrak{s})$ be the natural filtration. For every $d \in \mathbf{N}$, there exists a representation $\phi_{d}$ of $U(\mathfrak{s})$ acting on a finite dimensional Banach space $E_{d}$, such that $\operatorname{ker} \phi_{d} \cap U^{d}(\mathfrak{s})=0\left[\mathbf{2}\right.$, Theorem 2.5.7]. By Lie's Theorem, $\psi_{d}$, the restriction of $\phi_{d}$ to $U(\mathfrak{r})$, can be triangularized, and $\rho_{d}$, the restriction to $U(\mathfrak{n})$, will be strictly triangular.

Note that $\operatorname{ker} \rho_{d} \cap U^{d}(\mathfrak{n})=0$. Our problem is to combine the $\rho_{d}$ into a single, faithful representation. Let $y_{i}=e_{i i+1} \in \mathfrak{n}(1 \leq i \leq n-1)$. The $y_{i}$ generate $\mathfrak{n}$ as a Lie algebra. Let $Y_{i}^{d}=\rho_{d}\left(y_{i}\right) \in \mathcal{L}\left(E_{d}\right)$. Since the $Y_{i}^{d}$ are strictly triangular for a given $d$, we can arrange by a change of norm on $E_{d}$ to have $\left\|Y_{i}^{d}\right\| \leq 1(1 \leq i \leq n-1)$.

Received by the editors May 21, 1986.

1980 Mathematics Subject Classification (1985 Revision). Primary 46H99; Secondary 17 B35.

The author acknowledges support from the European Science Exchange Programme. 
Do this for every $d$, let $E$ be the $l^{1}$-direct sum of the $E_{d}$, and put $\rho=\rho_{1} \oplus \rho_{2} \oplus \cdots$ acting on $E$. Then $\left\|\rho\left(y_{i}\right)\right\|_{\mathcal{L}(E)} \leq 1$, and we get a map $U(\mathfrak{n}) \rightarrow \mathcal{L}(E)$ which is a faithful representation of $U(\mathfrak{n})$ by bounded operators on $E$.

\section{REFERENCES}

1. H. G. Dales, Norming nil algebras, Proc. Amer. Math. Soc. 83 (1981), 71-74.

2. J. Dixmier, Enveloping algebras, North-Holland, Amsterdam-New York-Oxford, 1977.

3. J. Esterle, Homomorphismes discontinus des algèbres de Banach commutatives séparables, Studia Math. 66 (1979), 119-141.

4. D. Gurarie, Banach uniformly continuous representation of Lie groups and algebras, J. Funct. Anal. 36 (1980).

5. J. L. Taylor, A general framework for a multi-operator functional calculus, Adv. Math. 9 (1972), 183-252.

School of Mathematics, The University, NewCastle Upon Tyne, NE1 7RU UNITED KINGDOM

Current address: Départment de Mathématiques, Université Libre de Bruxelles, 1050 Bruxelles, Belgium 